\title{
Potential Controlled Co-Ni Nanowires with Compositional Gradient
}

\author{
George Tepes, Maria Diana Vranceanu, Cosmin Mihai Cotrut, Dionezie Bojin
} University POLITEHNICA of Bucharest, Faculty of Materials Science and Engineering, Bucharest, 060042, Romania.

\author{
Ruxandra Vidu* \\ University of California, Davis, Department of Chemical Engineering and \\ Materials Science, Davis CA 95616, United States of America \\ Corresponding Author: rvidu@ucdavis.edu
}

\begin{abstract}
Co-Ni nanowires with composition gradient were grown by template assisted electrochemical deposition. Using PCTE membrane to grow nanowires makes the deposition of an alloy inside the pores more challenging because the growth is affected by the transport of the electroactive ions into the pores. A novel step-wise deposition-stripping strategy was used to grow NWs with controlled composition along the length of nanowire while maintaining constant the electrolyte composition. A detailed electrochemical analysis was performed to understand and control the Co stripping process, and to demonstrate the applicability of the proposed method. Redox reactions allow us to create custom deposition-stripping programs that enable us to better select and control the potential used to grow the nanowires of controlled composition. Samples were analyzed using scanning electron microscopy (SEM) and EDS. Quantitative analysis performed on samples has revealed that the designed stripping technique and selected potentials resulted in Co-Ni nanowires with up to 3 times more Ni then the samples obtained from similar electrolyte.
\end{abstract}

Keywords: nano, anomalous deposition, $\mathrm{Ni}, \mathrm{Co}$, stripping

\section{Introduction}

The interest in nanomaterials is motivated by their unique properties that can be applied to electronic devices, nanosensors, imaging devices and data storage. High versatility of nanostructures is due their physical properties, high surface to area ratio, and electronic properties [1-6].

Nanowires (NWs) with good magnetic properties and electrical conductivity are obtained from $\mathrm{Co}-\mathrm{Ni}$ alloys [2-12]. However, the control of the NW composition is challenging in the iron group alloys due to the anomalous deposition [9, 10, 12-14], which presents an obstacle to the straightforward codeposition of $\mathrm{Co}-\mathrm{Ni}$ alloys. Anomalous deposition occurs when the less noble of the two codepositing species deposits at an extremely higher concentration than the less noble species.

This paper aims to better control the chemical composition of Co-Ni NWs. In order to obtain the desired physicochemical properties, an advanced and improved control over the final NWs concentration is needed. Stripping technique can be used to restrict the anomalous deposition and to allow for a preferential oxidization of the less noble metal, which is Co in this case.

Generally speaking, to control the composition of $\mathrm{Co}-\mathrm{Ni}$ nanowires, the deposition may be performed in a single electrolyte containing Co and $\mathrm{Ni}$ ions $[1,2,8,9,11,12,15-21]$. Another way to control the deposition, which is less common, is to perform the deposition in alternating electrolytes containing either $\mathrm{Co}$ or $\mathrm{Ni}[4,6,22]$. Unlike the deposition in two electrolytes, the deposition in a single electrolyte results in an alloy whose composition can be modified by adjusting the electrolyte concentration $[1,2,8,9,11,12,15-21]$. This method allows for a better control of the composition by managing the deposition protocol (i.e. high frequency pulses, adjusting the deposition time, potential and rate).

Another advantage of this deposition in a single electrolyte is that segments of various compositions can be obtained. Alloy concentration in each 
segment is enforced by the concentrations of depositing ions in the electrolyte and the applied potential. The length of the segments is adjusted by changing the time of deposition at a certain potential. The main disadvantage of this method is that it is generally more difficult to obtain segments containing only one depositing element.

In the literature, the most common used ratio of $\mathrm{Co}: \mathrm{Ni}$ in the solution is $1: 2$, and the most common deposition potentials for $\mathrm{Co}$ and $\mathrm{Ni}$ are -0.4 and $1.5 \mathrm{~V}$, respectively $[2,9,12,23]$. However, Co and $\mathrm{Ni}$ show anomalous deposition that gives a different ratio of $\mathrm{Co}$ and $\mathrm{Ni}$ in the deposit [17-19]. Therefore, the compositional control in co-deposition is very important while the deposition in nanostructured template imposes additional challenges.

In this paper we applied pulse deposition because the relaxation times ("off time") allows for a more complex processes that may help us control the composition of the deposit. Relaxation times allow ions to diffuse to the electric double layer, thus preventing the depletion of depositing ions. Due to the difference in ion velocities, the relaxation time allows for $\mathrm{Ni}$ atoms to "relax" while Co atoms are oxidizing.

\section{Sample Preparation}

All samples and electrolytes were obtained using high purity materials and chemicals, and using a working protocol that helped to obtain high reproducible results. Specific protocols were developed for template preparation, sample preparation, electrochemical treatment and NWs growth.

To prepare the template for NWs growth, we first sputter Au on one side of the PCTE membrane. Next, the membrane was immersed in n-Butanol solution and sonicated for 5 minutes. After sonication, the template is placed with the gold side on a copper tape and sandwiched in between two isolating tapes. A small hole in the tape exposes the sample and allows the ions to reach the gold electrode through the template pores. These samples are used as Working Electrodes (WE) in a 3-electrode electrochemical cell that are connected to a potentiostat/galvanostat PARSTAT 4000 (Princeton Applied Research AMETEK, USA). A calomel electrode was use as a Reference Electrode and a platinum rod as a Counter Electrode.
To assure the reproducibility of the NWs results, an electrochemical treatment in $50 \mathrm{mM} \mathrm{H}_{2} \mathrm{SO}_{4}$ is conducted. This treatment consists of 2 steps: a Cyclic Voltammetry (CV) step that helps with the $\mathrm{Au}$ film cleaning, and a potentiostatic (PT) step. The main aim of this procedure is to clean the $\mathrm{Au}$ film surface and to favor $\mathrm{Au}$ atoms surface diffusion to result a flat surface [24-31].

\section{Experiments}

A series of CVs were conducted in order to understand the electrochemical behavior of $\mathrm{Au}$ in various electrolytes such as: blank solution $(40 \mathrm{~g} / 1$ $\left.\mathrm{H}_{3} \mathrm{BO}_{3}\right)$, Ni (44 g/l NiSO $\left.6 \mathrm{H}_{2} \mathrm{O}+40 \mathrm{~g} / \mathrm{l} \mathrm{H}_{3} \mathrm{BO}_{3}\right)$ and $\mathrm{Co}\left(20 \mathrm{~g} / 1 \mathrm{CoSO}_{4} \cdot 7 \mathrm{H}_{2} \mathrm{O}+40 \mathrm{~g} / \mathrm{l} \mathrm{H}_{3} \mathrm{BO}_{3}\right)$ electrolytes. Electrodeposition in pulses was performed using a PCTE membrane to grow Co-Ni NWs. The electrochemical behavior of $\mathrm{Au}$ in each of the 3 solutions was investigated to observe if there are any surface reactions in that potential range. For each solution, there were recorded 2 cycles.

Characterization of the Co-Ni NWs was performed using a scanning electron microscope equipped with an EDS. First the PCTE membrane is dissolved in a concentrated $\mathrm{Cl}_{2} \mathrm{CH}_{2}$ solution. Then, samples are washed with Milli-Q water and dried with $\mathrm{N}_{2}$.

\section{Results and Discussions}

\subsection{Electrochemical characterization}

CVs were performed in a blank solution (BS), $\mathrm{Co}$ and Ni electrolytes.

Figure 1a shows the $\mathrm{CV}$ curves of $\mathrm{Au}$ in blank solution (BS), where there are no depositions. Analysis of $\mathrm{CV}$ in $\mathrm{BS}$ show no faradaic reaction in the scanned potential range. Fig. 1 b,c shows the potentials that redox reactions start and end for each electrolyte, i.e. $\mathrm{Co}$ and $\mathrm{Ni}$ ion containing electrolytes. These potentials are then used to design an analytical program for obtaining a certain chemical composition gradient in nanowires.

The electrochemical parameters for nanowire growth were chosen based on the analysis of cyclic voltammetry. The CVs are obtained by scanning the potential between two selected values to study the redox reactions that may occur on the electrode in the selected electrolyte. We selected several 
potential (between $-1.1 \mathrm{~V}$ and $0,0.1$ and $0.2 \mathrm{~V}$ ) and scanning speed of 2,5 and $50 \mathrm{mV} / \mathrm{s}$, (for instance, Figure 1 presents only at $50 \mathrm{mV}$ ).

In $\mathrm{Ni}$ and $\mathrm{Co}$ electrolytes, as the applied potential moves to more negative values, the current increases due to the electrochemical reaction and deposition of atoms on of the working electrode surface. Figure 1 shows that Co reducing begins around $-0.58 \mathrm{~V}$ while $\mathrm{Ni}$ reduction begins around the $-0.37 \mathrm{~V}$.

After repeating each experiment twice to check the reproducibility the $\mathrm{CV}$ curves were used to determinate the potential steps for our designed experiments.


Figure 1. Cyclic voltammetry of Au in blank solution (1), Ni (b) and Co (c) ion containing solution at a sweep rate of $50 \mathrm{mV} / \mathrm{s}$.

\subsection{NWs growth}

We applied two electrochemical experiments to grow NWs with custom compositional gradient on the longitudinal axis. The electrochemical experiments were set one with stripping off (OFF) (Figure 2) and one with stripping on (ON) (Figure 3). After the NWs growth, the samples were prepared for SEM and EDS analysis.

For both OFF and ON, the final pulse is set to allow for Co deposition, so that at the top of the NWs, Co concentration is almost the same. Figures 2.a and 3.a present the charts of the applied potentials and of the recorded current during the NWs growth.

\subsection{Scanning electron microscopy}

SEM images that were taken on the Co-Ni NWs samples (Figure 2, b) - d) and Figure 3 b) - d)) offer valuable information on NWs dimension and surface topography. At a first glance, SEM images show that the NWs obtained with ON are shorter than the ones obtained with OFF, at a same growth time. SEM measurements showed that the NWs obtained in stripping ON conditions are $2.3 \pm 0.3$ $\mu \mathrm{m}$ long compared with OFF that have grown in to mushrooms ( $>6 \mu \mathrm{m})$. Mushroom morphology is a common morphology of overgrown nanowires that continue to grow after they reach the surface.

NWs diameter is $150 \pm 20 \mathrm{~nm}$ which is $40 \%$ larger than the measured membrane pore diameter, i.e. $113.7 \pm 25 \mathrm{~nm}$. This difference between the NW diameter and the diameter of the pore has been previously reported [23] as being caused as a result of swelling of pores during the hydrophilic treatment in n-Butanol.

To better understand the chemical composition profile obtained in NWs, EDS analysis was conducted on samples obtained in both sets of experiments. Results are shown in Table 1 and 2. Each NW was scanned and measured in 3 points, at the ends and in the middle. 

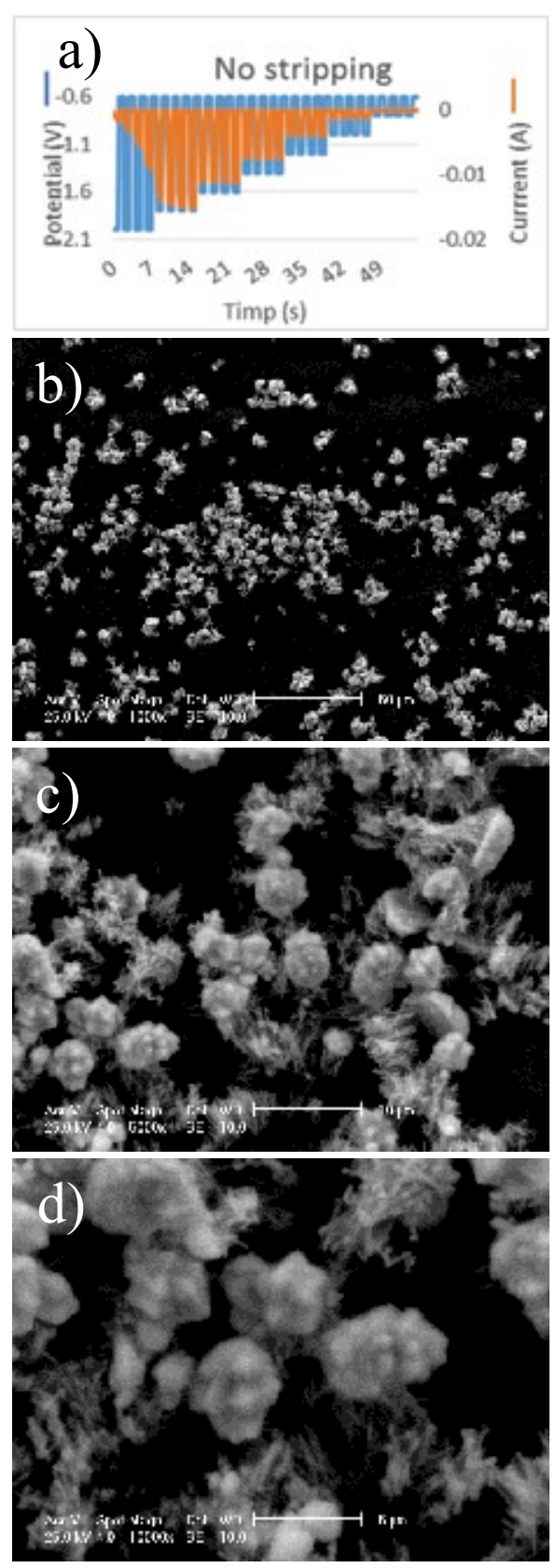

Figure 2. a-Chart of the applied potentials (blue) along with the recorded current (orange) vs time;

b, c, d-SEM images of NWs obtained with stripping OFF.
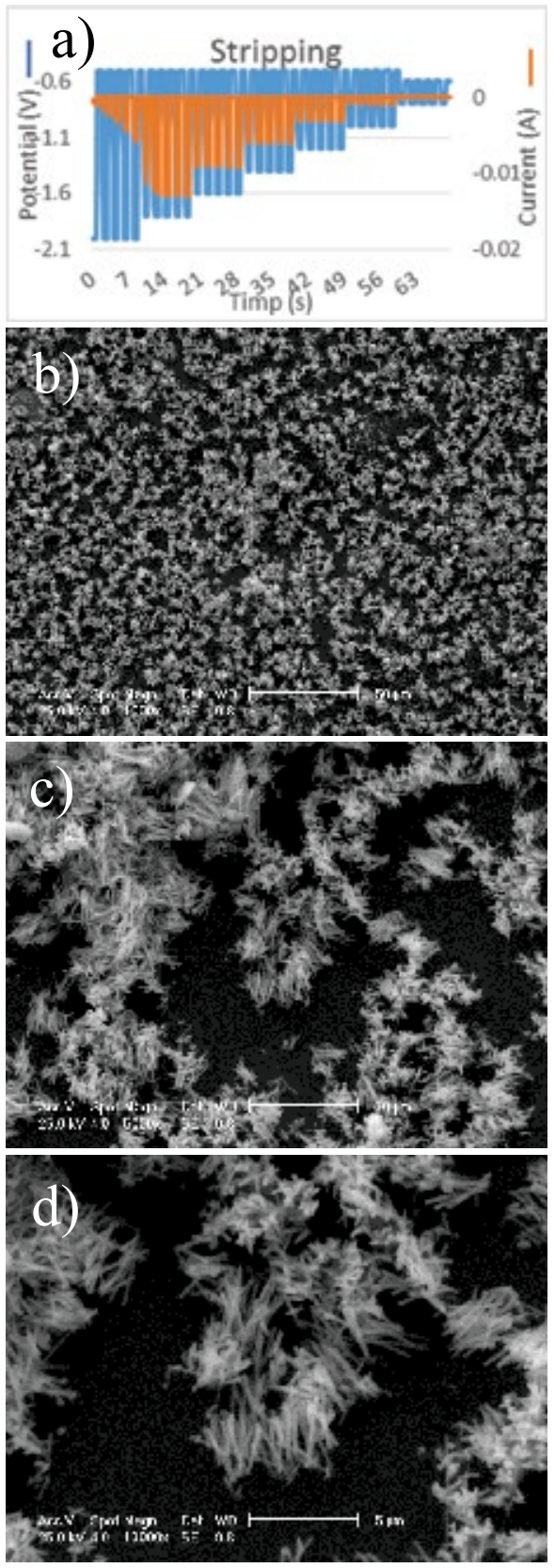

Figure 3. a - graphical representation of the applied potentials (blue) along with the recorded current (orange) vs time;

b, c, d-SEM images of NWs obtained with stripping $\mathrm{ON}$. 
Table 1: Stripping ON

\begin{tabular}{ccc}
\hline Experiment No. & $\mathrm{Co}$ & $\mathrm{Ni}$ \\
\hline 1 & 20.45 & 79.55 \\
2 & 33.67 & 66.33 \\
3 & 69.69 & 30.31
\end{tabular}

Table 2: Stripping OFF

\begin{tabular}{ccc}
\hline Experiment No. & $\mathrm{Co}$ & $\mathrm{Ni}$ \\
\hline 5 & 28.71 & 71.29 \\
6 & 36.64 & 63.36 \\
7 & 67.93 & 32.07
\end{tabular}

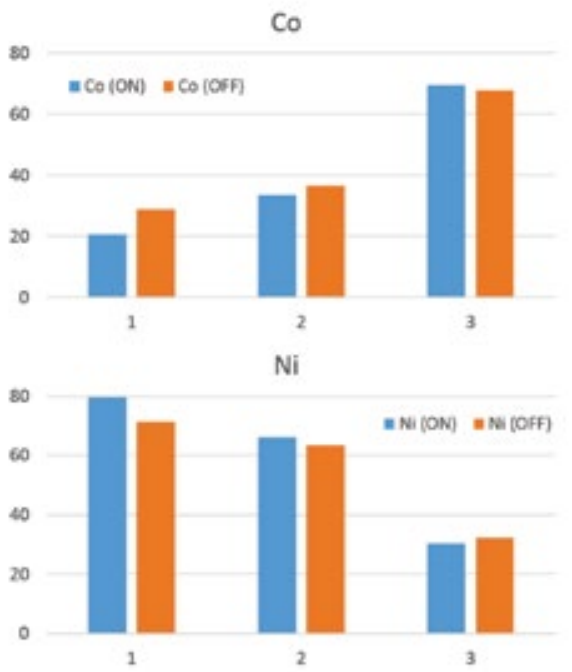

Figure 4. Comparison of $\mathrm{Co}$ and $\mathrm{Ni}$ concentrations measured in NWs

According to EDS results, NWs sample that are obtained using $\mathrm{ON}$ presents an increased $\mathrm{Ni}$ concentration at one end, i.e. $79 \%$, compared to the NWs obtained with OFF, i.e. $71 \%$. In the literature, using electrolytes with much higher $\mathrm{Ni}$ :Co ratio, such as 21:1/Ni:Co (i.e. 1,250 $\mathrm{M} \mathrm{NiCl}_{2} \cdot 6 \mathrm{H}_{2} \mathrm{O}$ and $0,06 \mathrm{M} \mathrm{CoCl}_{2} \cdot 6 \mathrm{H}_{2} \mathrm{O}$ ) [23] or $30: 1 / \mathrm{Ni}$ :Co (i.e. 300 $\mathrm{g} / 1 \mathrm{NiSO}_{4} \cdot 7 \mathrm{H}_{2} \mathrm{O}$ and $\left.10 \mathrm{~g} / \mathrm{l} \mathrm{CoSO}_{4} \cdot 7 \mathrm{H}_{2} \mathrm{O}\right)$ [4], similar Ni concentration difference in NWs was observed.

Additionally, the EDS measurements show that $\mathrm{Ni}$ concentration decreases along the length of the NWs in both ON and OFF growth situations. These results may be due to the difference in diffusion rates of $\mathrm{Co}$ and $\mathrm{Ni}$ at more positive potentials, where Co has higher diffusion rate than $\mathrm{Ni}$.

\section{Conclusions}

Ni-rich NWs have been obtained using same $\mathrm{Co}$ :Ni ratio as reported in the literature. Using the same electrolyte that Prida et all $[2,9]$ reported to obtain Co reach (Ni poor NWs $(46 \%)$ ), we have obtained Co poor NW (Ni reach NWs (79\%)) using a pulse electrochemical schedule.

The composition of the NWs as measured by SEM/EDS is variable along the longitudinal axes. The pulse electrochemical techniques used in these experiments resulted in Ni reach NWs obtained in a low concentration $\mathrm{Ni}$ electrolyte. This pulse technique can be used to overcome the anomalous deposition that may appear in Ni-Co electrolytes and opens new ways to control the deposition and growth rate.

\section{Acknowledgment}

The work has been funded by the Sectorial Operational Program Human Resources Development 2007-2013 of the Ministry of European Funds through the Financial Agreement POSDRU/159/1.5/S/134397.

\section{References}

1. Chen, M., C.-L. Chien, and P.C. Searson, Potential Modulated Multilayer Deposition of Multisegment $\mathrm{Cu} / \mathrm{Ni}$ Nanowires with Tunable Magnetic Properties. Chemistry of Materials, 2006. 18(6): p. 1595-1601.

2. García, J., et al., Template-assisted $\mathrm{Co}-\mathrm{Ni}$ alloys and multisegmented nanowires with tuned magnetic anisotropy. physica status solidi (a), 2014. 211(5): p. 1041-1047.

3. Hussain, M., et al., Fabrication and temperature dependent magnetic properties of $\mathrm{Ni}-\mathrm{Cu}-\mathrm{Co}$ composite nanowires. Physica B: Condensed Matter, 2015. 475: p. 99-104.

4. Pereira, A., et al., A soft/hard magnetic nanostructure based on multisegmented CoNi nanowires. Physical Chemistry Chemical Physics, 2015. 17(7): p. 5033-5038.

5. Vivas, L., et al., Magnetic anisotropy in CoNi nanowire arrays: analytical calculations and experiments. Phys Rev B, 2012. 85: p. 035439.

6. Wang, H., et al., Effect of sub-layer thickness on magnetic and giant magnetoresistance properties of $\mathrm{Ni}-\mathrm{Fe} / \mathrm{Cu} / \mathrm{Co} / \mathrm{Cu}$ 
multilayered nanowire arrays. Chinese Journal of Chemical Engineering, 2015. 23(7): p. 1231-1235.

7. Shahzad Khan, B., et al., Effect of workfunction on the growth of electrodeposited $\mathrm{Cu}, \mathrm{Ni}$ and $\mathrm{Co}$ nanowires. Materials Letters, 2014. 137: p. 13-16.

8. Vilana, J., E. Gómez, and E. Vallés, Electrochemical control of composition and crystalline structure of CoNi nanowires and films prepared potentiostatically from a single bath. Journal of Electroanalytical Chemistry, 2013. 703: p. 88-96.

9. Prida, V., et al., Electroplating and magnetostructural characterization of multisegmented Co54Ni46/Co85Ni15 nanowires from single electrochemical bath in anodic alumina templates. Nanoscale Research Letters, 2013. 8(1): p. 263.

10. Vazquez-Arenas, J. and M. Pritzker, Steady-state model for anomalous $\mathrm{Co}-\mathrm{Ni}$ electrodeposition in sulfate solutions. Electrochimica Acta, 2012. 66: p. 139-150.

11. Hamrakulov, B., et al., Electrodeposited $\mathrm{Ni}, \mathrm{Fe}$, $\mathrm{Co}$ and $\mathrm{Cu}$ single and multilayer nanowire arrays on anodic aluminum oxide template. Transactions of Nonferrous Metals Society of China, 2009. 19, Supplement 1: p. s83-s87.

12. Ghahremaninezhad, A. and A. Dolati, A study on electrochemical growth behavior of the $\mathrm{Co}-\mathrm{Ni}$ alloy nanowires in anodic aluminum oxide template. Journal of Alloys and Compounds, 2009. 480(2): p. 275-278.

13. Chung, C.K. and W.T. Chang, Effect of pulse frequency and current density on anomalous composition and nanomechanical property of electrodeposited $\mathrm{Ni}-\mathrm{Co}$ films. Thin Solid Films, 2009. 517(17): p. 4800-4804.

14. Oriňáková, R., et al., Influence of $\mathrm{pH}$ on the electrolytic deposition of $\mathrm{Ni}-\mathrm{Co}$ films. Thin Solid Films, 2008. 516(10): p. 3045-3050.

15. Dolati, A. and S.S. Mahshid, A study on the kinetics of Co$\mathrm{Ni} / \mathrm{Cu}$ multilayer electrodeposition in sulfate solution. Materials Chemistry and Physics, 2008. 108(2-3): p. 391-396.

16. Nasirpouri, F., et al., GMR in multilayered nanowires electrodeposited in track-etched polyester and polycarbonate membranes. Journal of Magnetism and Magnetic Materials, 2007. 308(1): p. 35-39.

17. Rohan, J.F., et al., Coaxial metal and magnetic alloy nanotubes in polycarbonate templates by electroless deposition. Electrochemistry Communications, 2008. 10(9): p. 1419-1422.

18. Azarian, A., et al., Field emission of Co nanowires in polycarbonate template. Thin Solid Films, 2009. 517(5): p. 1736-1739.
19. Mathe, V.L. and A.D. Sheikh, Magnetostrictive properties of nanocrystalline Co-Ni ferrites. Physica B: Condensed Matter, 2010. 405(17): p. 3594-3598.

20. Ohgai, T., Magnetoresistance of Nanowires Electrodeposited into Anodized Aluminum Oxide Nanochannels, X. Peng, Editor. 2012, InTech. p. 101-125.

21. Monzon, L.M.A., et al., Fabrication of multisegmented magnetic wires with micron-length copper spacers. Electrochemistry Communications, 2013. 36: p. 96-98.

22. Maleak, N., et al., Fabrication and magnetic properties of electrodeposited $\mathrm{Ni} / \mathrm{Cu}$ nanowires using the double bath method. Journal of Magnetism and Magnetic Materials, 2014. 354: p. 262-266.

23. Hansal, W.E.G., et al., Pulse reverse plating of $\mathrm{Ni}-\mathrm{Co}$ alloys: Deposition kinetics of Watts, sulfamate and chloride electrolytes. Electrochimica Acta, 2006. 52(3): p. 1145-1151.

24. Vidu, R. and S. Hara, Surface alloying at the Cd/Au(100) interface in the upd region. Electrochemical studies and in situ EC-AFM observation. Journal of Electroanalytical Chemistry, 1999. 475(2): p. 171-180.

25. George Tepes*, A.A.M., Maria Diana Vranceanu, Cosmin Mihai Cotrut, Dionezie Bojin, Victor Kuncser, Ruxandra Vidu. Influence of the electrochemical treatment on the magnetic properties of nanowires. in American Romanian Academy. 2015. National Institute of Nuclear Physics, Frascati, Roma.

26. Ruxandra Vidu, S.H., In situ electrochemical atomic force microscopy study on $\mathrm{Au}, 100$.../Cd interface in sulfuric acid solution. American Vacuum Society.

27. K. Kubo*, N.H., S. Hara, Decay of nano-islands on Au(1 00 0 electrode in sulfuric acid solution with $\mathrm{Cl}$ anions. Applied Surface Science, 2004. 237: p. 301-305.

28. Nobumitsu HIRAI, H.O.a.S.H., In Situ Electrochemical Atomic Force Microscopy with Atomic Resolution of Fe(110) in Sodium Sulfate Aqueous Solution. ISIJ International, 2000. 40(7): p. 702 - 705.

29. Nobumitsu Hirai, H.T., Shigeta Hara, Enhanced diffusion of surface atoms at metalrelectrolyte interface under potential control. Applied Surface Science, 1998: p. 506-511.

30. Nobumitsu Hirai, K.-i.W., Akiko Shiraki, and Shigeta Hara, In situ atomic force microscopy observation on the decay of small islands on Au single crystal in acid solution. Journal of Vacuum Science \& Technology B, 2000. 18(7).

31. Nobumitsu Hirai, M.Y., Toshihiro Tanaka, Shigeta Hara, Decay of nano-islands on the surface of a Au(111) electrode in contact with sulfuric acid solution. Science and Technology of Advanced Materials 5, 2003: p. 115-118. 